\title{
Increase in hospital admissions for torsion of testis
}

\author{
M NELMS AND D COGGON \\ From the MRC Environmental Epidemiology Unit, University of Southampton, Southampton General \\ Hospital, Southampton SO9 $4 X Y$
}

SUmmary Data from Hospital Activity Analysis (HAA) for the Wessex Health Region show a $70 \%$ increase in hospital admissions for torsion of the testis between 1971 and 1982 . The frequency of emergency admission leading to orchidopexy doubled, but there was no increase in the rate of emergency admission followed by orchidectomy. Detailed investigation of records from Southampton hospitals suggests that the trend cannot be explained by errors in the ascertainment of cases by HAA. The rise in admissions could result from a greater awareness of the diagnosis among general practitioners leading to more frequent referral of mild cases, but it seems more likely that it reflects a real increase in the incidence of the disorder.

Torsion of the testis is a surgical emergency. It typically presents as a painful swelling of the testis associated with pain in the groin or lower abdomen, nausea, and vomiting. If treatment is delayed the testis is likely to be lost through infarction. Thus when the diagnosis is suspected, immediate surgical referral is the rule.

Testicular torsion was first distinguished as a separate diagnostic category in the eighth revision of the International Classification of Diseases (ICD). Analysis of data from the Hospital In-patient Enquiry (HIPE) has indicated a doubling in hospital admissions for torsion of the testis in England and Wales during the period $1968-80 .{ }^{1}$ This rise could be due to artefacts in the ascertainment of cases by HIPE; to a change in clinical practice with a trend to the more frequent admission of mild cases or perhaps to multiple admissions of individual patients; or it could reflect a real increase in the incidence of the disease. We have used data from Hospital Activity Analysis (HAA) for the Wessex Health Region to explore these possibilities further.

\section{Method}

We obtained from Wessex Regional Health Authority abstracts of the HAA records of all hospital patients in the region who were discharged or died during the period 1971-82 with a main or subsidiary diagnosis of torsion of the testis (ICD $8=607 \cdot 7$, ICD $9=608 \cdot 2$ ). The information was supplied on magnetic tape and, for each admission, included the date of birth of the patient, the type of admission (emergency or planned), the date of discharge, and a list of any operations performed. The health authority also provided us with annual population estimates for the region, and from these we derived discharge rates directly standardised for age. The rates were calculated for the age range 5-34 (in which most cases occur) and were standardised using five year age groups with the 1971 population of England and Wales as a reference.

To assess the validity of the HAA data we carried out a more detailed investigation in the city of Southampton where virtually all acute surgical disease is referred to the Southampton group of hospitals. We obtained HAA listings of all patients discharged from Southampton hospitals during 1971-81 with a diagnosis of testicular torsion, and we reviewed the case notes of those who were aged 5-34 and resident in Southampton to check the accuracy of the recorded discharge diagnosis. We also examined the notes of patients treated for "other specified male genital anomalies" (ICD $8=752 \cdot 8$, ICD $9=752 \cdot 8$ ) since this diagnostic category includes torsion of the hydatid of Morgagni, a diagnosis which might be confused with true testicular torsion. In addition, we scanned operation books at the hospitals to identify all patients aged 5-34 who underwent surgery to the testis or scrotum during the years 1971-74. We then reviewed the notes of these patients in a search for any further cases of testicular torsion in Southampton residents which had not been ascertained by HAA.

\section{Results}

The figure shows trends in discharge rates for torsion of the testis in Wessex according to the type of 
admission and the surgical operation performed. Altogether, 1460 hospital admissions were attributed to testicular torsion during the period 1971-82. As in the whole of England and Wales, the discharge rate increased substantially during the 1970s. Furthermore, the rise was due largely to an increase in emergency admissions. The frequency of emergency admission followed by orchidopexy doubled between 1971 and 1982, but there was no increase in the rate of emergency admission followed by orchidectomy.

In the validation exercise carried out in Southampton we were able to trace hospital notes for $96 \%$ of the patients identified from HAA records and operation books. Altogether, 144 patients were recorded by HAA as receiving treatment for torsion of the testis during the period 1971-81, and we confirmed the diagnosis for 124 of these patients (see table). Among the 17 cases misclassified as testicular torsion, torsion of the hydatid of Morgagni was the most common diagnosis (10 patients). The rate of misclassification was higher in the later years of the study period, but this did not explain the rise in recorded admissions. The notes of three patients with an HAA diagnosis of testicular torsion could not be located.

No torsions of the testis were found among the patients with admissions attributed to "other specified male genital anomalies". Examination of the records of patients who underwent testicular or scrotal surgery during the period 1971-74 revealed five additional cases of torsion of the testis which had

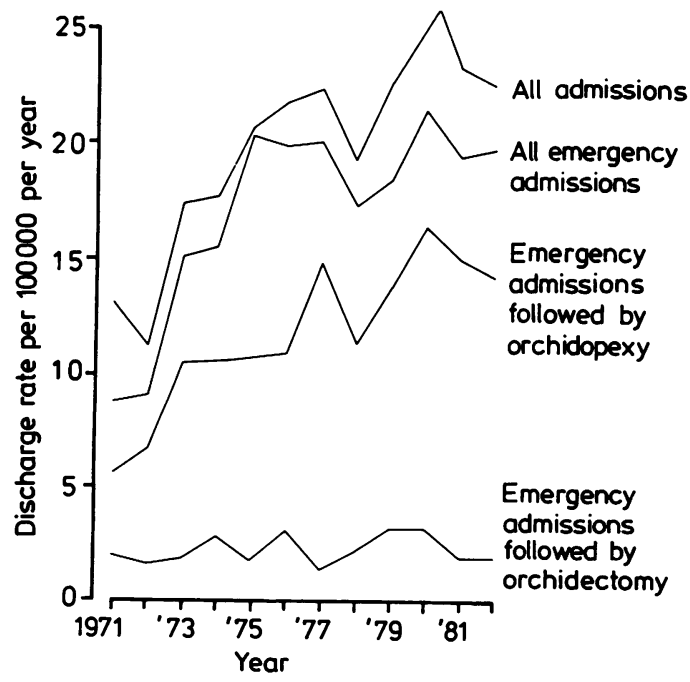

Age-standardised discharge rates for torsion of the testis in males aged 5-34, Wessex Health Region, 1971-82.
Accuracy of HAA diagnoses of testicular torsion in residents of Southampton aged 5-34

\begin{tabular}{llll}
\hline & $\begin{array}{l}\text { Patients with testicular } \\
\text { torsion correctly } \\
\text { recorded by HAA }\end{array}$ & $\begin{array}{l}\text { Patients with other } \\
\text { diagnoses incorrectly } \\
\text { recorded as testicular } \\
\text { torsion }\end{array}$ & $\begin{array}{l}\text { Patients whose } \\
\text { hospital notes could } \\
\text { not be found }\end{array}$ \\
\hline 1971 & 11 & 0 & 0 \\
1972 & 4 & 1 & 0 \\
1973 & 7 & 1 & 0 \\
1974 & 14 & 0 & 0 \\
1975 & 6 & 2 & 0 \\
1976 & 11 & 2 & 0 \\
1977 & 13 & 3 & 1 \\
1978 & 10 & 2 & 1 \\
1979 & 12 & 2 & 0 \\
1980 & 15 & 3 & 0 \\
1981 & 17 & 1 & 1 \\
\hline
\end{tabular}

*If a patient was admitted to hospital on several occasions, only the first admission has been counted.

not been correctly ascertained by HAA. Including these cases, a total of 41 Southampton residents in the age range 5-34 were treated for torsion of the testis during 1971-74. This compares with a minimum of 54 patients (the cases correctly recorded by HAA) treated between 1978 and 1981 .

\section{Discussion}

Our analysis of HAA data for torsion of the testis in Wessex shows a similar trend to that demonstrated for the whole of England and Wales from HIPE. ${ }^{1}$ The increase in recorded discharges is substantial and as such is unlikely to result from random fluctuation. It could, however, be due to artefacts in the ascertainment of cases by HAA with a tendency to under-recording in the early 1970 s or to over-recording in recent years. Previous attempts to validate HAA data have exposed serious deficiencies in the ascertainment of admission for fracture of the proximal femur ${ }^{2}$ and in the documentation of surgical procedures such as splenectomy and colectomy. ${ }^{3}$ However, ascertainment may be more reliable for conditions such as torsion of the testis where the differential diagnosis is limited and admission is usually short and uncomplicated.

Our study in Southampton shows that between 1971 and 1981,17 patients were misclassified as having torsion of the testis by HAA. However, the error rate did not increase appreciably over the period studied. Nor did our search of operation records point to any major under-ascertainment of cases during the early years of the study. It is unlikely that many patients with a diagnosis of testicular torsion will have escaped surgery, and operation records are therefore a reasonable standard against which to assess the accuracy of HAA. After 
allowance for the errors which we detected in HAA data for Southampton, a rise in hospital admissions for testicular torsion was still apparent. Forty one patients were treated for torsion during 1971-74 and at least 54 between 1978 and 1981 . The true figure for $1978-81$ is likely to be somewhat higher given that the notes of two patients with HAA diagnoses of testicular torsion (one in 1978 and one in 1981) could not be found, and further cases may have been missed by HAA. The male population of Southampton in the age range 5-34 was virtually the same in each of the four-year periods.

Further evidence that admission rates for testicular torsion have increased comes from the analysis of a case series from Bristol, ${ }^{4}$ although in that study the population at risk was not clearly defined.

Given that the rise in admission rates for torsion is real, it could be due to an increase in the incidence of the disorder or to a change in its clinical management. It is unlikely to result from more frequent readmission of cases since the trend is apparent in our analysis for Southampton where we counted patients rather than hospital episodes. Furthermore, the HAA data for Wessex show an increase in the rate of emergency admission followed by orchidopexy, a sequence of events which is unlikely to befall an individual more than once.

The possibility remains that general practitioners are becoming more aware of testicular torsion and its consequences and have lowered their threshold for referring suspected cases to hospital. That the rate of emergency admission followed by orchidectomy has not gone up in parallel with other emergency admissions for torsion suggests that the spectrum of disease now arriving in hospital is more benign than in the past. This could be because more mild or doubtful cases are being referred, or it may be that patients are being referred earlier in the course of their illness at a time when the testis is still salvageable. Within the series of patients identified in Southampton there was no indication that patients presenting to hospital in the early years of the study had longer histories of symptoms than those admitted more recently. However, the number of cases is too small to be firm on this point. It seems unlikely that greater awareness on the part of general practitioners could on its own explain an increase in admissions of the magnitude that has been observed.

If the incidence of testicular torsion is really increasing, the reason is unclear. Little is known about the aetiology of the disorder save that it is usually associated with an anatomical abnormality in which the testis is suspended by a mesentery and lies horizontally (or in extreme cases is inverted) within the scrotum. ${ }^{5}$ We can find no reports of the prevalence of horizontal lie in the generai population, but an increase in its occurrence might explain the trends in testicular torsion. The malformation arises at an early stage of fetal development before the testis has descended from its original site in the abdomen. Recently, Chilvers and her colleagues have reported an apparent doubling in the frequency of undescended testis in England and Wales during the period $1962-81 .^{6}$ While relatively few torsions occur in undescended testes, it is possible that a common aetiological factor is causing a rise in the incidence of both maldescent and horizontal lie of the testis, and perhaps also of other congenital abnormalities of the male urogenital tract. National statistics suggest a rise also in the incidence of hypospadias and hydrocele. ${ }^{7}$

Another possibility is that the circumstances which precipitate torsion in susceptible individuals are becoming more common. Various factors are suspected of triggering testicular torsion including physical exercise,${ }^{8}$ trauma,${ }^{8}$ sexual activity, ${ }^{8}$ and cold weather. ${ }^{9}$ However, the evidence implicating these environmental influences is far from conclusive, and examination of case series suggests that none of them is responsible for more than a small proportion of cases. ${ }^{4810}$ It follows that none of the factors could on its own explain a large increase in the incidence of testicular torsion.

Whatever the reason for the rise in hospital admissions for testicular torsion, further surveillance of the trend is indicated. If more boys and young men are undergoing surgery for torsion in the absence of any change in disease occurrence we should ask whether this is appropriate. If the disorder is really becoming more common we should find out why. More information is needed about the prevalence of horizontal lie in the general population. In addition, there is a clear need for a case-control study of testicular torsion. Such an investigation should look in particular at factors such as maternal weight and use of oral contraceptives which by altering the endocrine environment of the fetus might predispose to abnormalities of the developing urogenital tract and so explain a rising incidence of torsion.

We thank Mr M Slattery, who provided us with the HAA data for Wessex, Mr P Norman, who helped us with the computing, and the surgeons at Southampton hospitals who allowed us access to their notes: Mr J D Atwell, Mr A Chant, Mr N Freeman, Mr J Jenkins, Mr S J Karran, Mr F McGinn, Mr C Smart, Mr H Steer, and Mr J Webster.

\section{References}

${ }^{1}$ Coggon D, Nelms $M$. Incidence of testicular abnormalities. Lancet 1984: ii: 747. 
${ }^{2}$ Rees JL. Accuracy of Hospital Activity Analysis data in estimating the incidence of proximal femoral fracture. Br Med J 1982; 284: 1856-7.

${ }^{3}$ Whates PD, Birzgalis AR, Irving M. Accuracy of Hospital Activity Analysis operation codes. Br Med J 1982; 284: 1857-8.

${ }^{4}$ Williamson RCN. Torsion of the testis and allied conditions. Br J Surg 1976; 63: 465-76.

${ }^{5}$ Scorer CG, Farrington GH. Congenital deformities of the testis and epididymis. London: Butterworths, 1971.

${ }^{6}$ Chilvers C, Pike MC, Forman D, Fogelman $K$, Wadsworth MEJ. Apparent doubling of frequency of undescended testis in England and Wales in 1962-81. Lancet 1984: ii: 330-2.

${ }^{7}$ Matlai P, Beral V. Trends in congenital malformations of external genitalia. Lancet 1985: i: 108.

${ }^{8}$ Abeshouse BS. Torsion of the spermatic cord; report of three cases and review of the literature. Urol Cutan Rev 1936; 40: 699-714.

${ }^{9}$ Shukla RB, Kelly DG, Daly L, Guiney EJ. Association of cold weather with testicular torsion. Br Med J 1982; 285: 1459-60.

${ }^{10}$ Angell JC. Torsion of the testicle: a plea for diagnosis. Lancet 1963: i: 19-21. 